American Journal of Biochemistry and Biotechnology 2 (4): 146-147, 2006

ISSN 1553-619X

(c) 2006 Science Publications

\title{
An Alternative to the Traditional Western Blot For Recombinant His-tagged Proteins
}

\author{
Justin B. Morgenthaler, Jason R. Stephenson, Jon A. Friesen and Marjorie A. Jones \\ Department of Chemistry, Illinois State University, Normal, IL. 61790-4160
}

\begin{abstract}
A rapid, inexpensive and sensitive alternative to the traditional Western Blot is presented here. This method is very useful for detection of recombinant 6-his tagged proteins.
\end{abstract}

Key words: Recombinant his-tagged proteins, electrophoresis

\section{INTRODUCTION}

The use of the immunoblot technology, also know as the Western blot ${ }^{[1]}$, has been a great asset in the rapidly expanding area of molecular biology, especially coupled to the technology for expression of recombinant proteins. However, there are several limitations in this technology. Western blots have traditionally required the use of an antibody (or antiserum) to detect a specific protein. This antibody usually has been applied after the proteins have been blotted onto a support, commonly nitrocellulose paper, from a denaturing polyacrylamide gel used to separate the proteins by apparent molecular weight. This blotting requires about 1 hour when done by application of a current or overnight when using a semidry blotting technique. In both blotting methods, buffers as well as other reagents and materials are required. The advent of the six-histidine (6-his) tag technology, in which 6 histidines are added to the code of the protein being expressed, allows for proteins to be easily and rapidly purified using either nickel or cobalt column resins ${ }^{[2]}$. This technology has greatly increased the efficiency of the targeted protein isolation. Recently, a reagent with nickel attached to the horseradish peroxidase (HRP) detection system has been marketed (Pierce) for use with the Western blots to eliminate the need for antiserum. The nickel HRP reagent (HisProbe ${ }^{\mathrm{TM}}$ HRP) binds to the 6-his tagged protein with high affinity and thus can be used in Western blot analysis. We now report an improvement on this methodology in which we carry out Sodium Dodecyl Sulfate-Polyacrylamide Gel Electophoresis (SDS-PAGE) and then probe the electrophoretogram directly with the HisProbe ${ }^{\mathrm{TM}}$ HRP, thus eliminating the time and expense of the blotting steps.

The protein used was human recombinant, coproporphyrinogen oxidase (copro'gen oxidase; E.C. 1.3.3.3) which contains a 6-his tag and has an approximate molecular weight of $37,500^{[2]}$. The HisProbe $^{\mathrm{TM}}$ HRP was obtained from Pierce (Rockford, IL). The working solution of the HisProbe ${ }^{\mathrm{TM}}$ HRP was prepared following the procedure provided by Pierce
(Pierce 2006). Stock probe samples were frozen at $-80^{\circ}$ $\mathrm{C}$ in $10 \mu \mathrm{L}$ aliquots. The wash buffer TBST, $(25 \mathrm{mM}$ Tris, $0.15 \mathrm{M} \mathrm{NaCl}, \mathrm{pH} 7.6,0.05 \%$ (v/v) Tween ${ }^{\circledR}-20$ ) as well as blocking buffer (25 mg BSA per ml of TBST) were prepared using the procedure provided by Pierce (Pierce 2006). SDS-PAGE was performed using the method of Laemmli ${ }^{[3]}$. After electrophoresis of the proteins, the gels were washed twice for 30 minutes each with TBST buffer to remove residual SDS. After the TBST washes, incubation for an hour with $10 \mu \mathrm{L}$ of HisProbe ${ }^{\mathrm{TM}} \mathrm{HRP}$ in $50 \mathrm{ml}$ of blocking buffer was done. Once the hour had past, the HisProbe ${ }^{\mathrm{TM}}$ HRP solution was then removed and the gel was washed twice for fifteen minutes each with TBST buffer. After the final TBST wash, the developing process was carried out using the AEC Chromogen Kit from Sigma (St. Louis, MO). After removal of the TBST buffer, $8 \mathrm{ml}$ of deionized water, two drops of Acetate buffer $(60 \mu \mathrm{L})$, two to three drops $(60-90 \mu \mathrm{L})$ of AEC Chromogen and two to four drops $(60-120 \mu \mathrm{L})$ of $30 \%$ hydrogen peroxide were added to the gel and incubated until a satisfactory pink color development was obtained.

Figure 1a is a representative SDS-PAGE (12\% gel) of copro'gen oxidase, (approximate MW of 37,500) with different concentrations of protein in each well, stained with the HisProbe ${ }^{\mathrm{TM}}$ HRP. Figure $1 \mathrm{~b}$ is the same gel post-stained with Coomassie blue. Figure 1c is a replicate gel that was first blotted onto nitrocellulose then probed with the nickel reagent. In each case, the HisProbe ${ }^{\mathrm{TM}}$ HRP reagent detected the protein over a 20 fold range corresponding to the same range that was detectable by the Coomassie blue and Western blot procedure. The data show that the transfer to nitrocellulose is not necessary using this nickel reagent and that we were able to detect protein bands as low as $0.9 \mathrm{ug}$. To test specificity of the HisProbe ${ }^{\mathrm{TM}} \mathrm{HRP}$, pure copro'gen oxidase as well as bacterial cell lysate containing this recombinant enzyme were loaded on the gel. Rainbow molecular weight markers by Amersham Biosciences as well as Promega Broad Range SDSPAGE standards were also loaded. Figure 2a shows the SDS-PAGE with the HisProbe ${ }^{\mathrm{TM}}$ HRP, $2 b$ is the gel shown in 2a post-stained with Coomassie blue and $2 \mathrm{c}$ is

Corresponding Author: $\quad$ Marjorie A. Jones, Department of Chemistry, Campus Box 4160, Illinois State University, Normal, IL. 61790-4160 

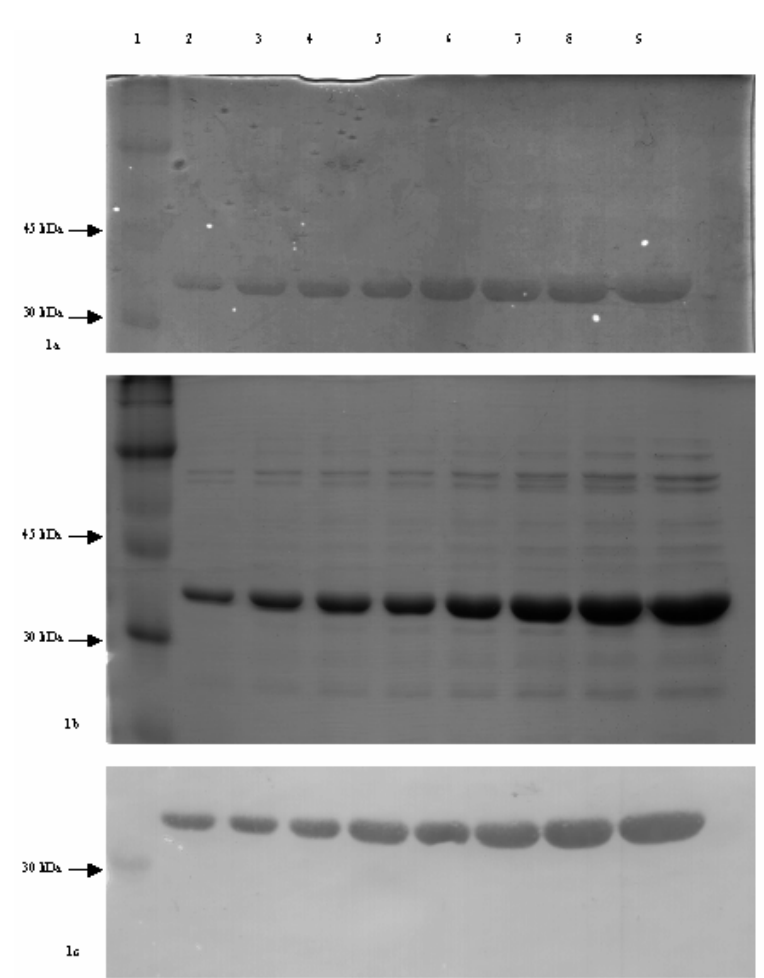

Fig. 1: (a) SDS-PAGE with HisProbe ${ }^{\mathrm{TM}}$ HRP; (b) Post staining of gel (a) with Coomassie blue; (c) Nitrocellulose Western Blot using the HisProbe $^{\mathrm{TM}}$ HRP. Lane 1: Molecular weight standard in $\mathrm{kDa}$ Lanes 2-9: Copro'gen oxidase in increasing amounts of protein per lane $(0.9$, $1.8, \quad 2.7, \quad 4.5, \quad 6.3, \quad 9.0, \quad 13.5, \quad 18.0 \mu \mathrm{g}$, respectively)

the traditional Western blot using the HisProbe ${ }^{\mathrm{TM}}$ HRP. As shown in Figure 2a, only the 6-his tagged protein was detected by the HisProbe ${ }^{\mathrm{TM}}$ HRP reagent (lane 4) and this is the same band detected in the traditional Western blot (Fig. 2c). The various protein bands shown in Fig. 2b thus were only detected by the Coomassie blue stain.

When comparing the data from our method to the traditional Western blot, it was found that our method was able to detect protein over the same concentration range as the Western blot (Fig. 1). Also, the method is specific for the 6-his tagged proteins (Fig. 2). However, traditional Western blots cost approximately 10 to 20 dollars more per gel than the non-blot method developed in our lab. This method also affords a researcher the ability to stain the gel with Coomassie blue after the probe has been added, something that cannot be done using nitrocellulose paper. In addition to being more cost effective and versatile, our method also takes at least 2 hours less time because the proteins do not have to be blotted onto the nitrocellulose paper. Our method is very simple to do, is rapid and has high reproducibility and high specificity making it a great alternative to the traditional Western blot when using the HisProbe ${ }^{\mathrm{TM}}$ HRP reagent with the 6-his tagged proteins.
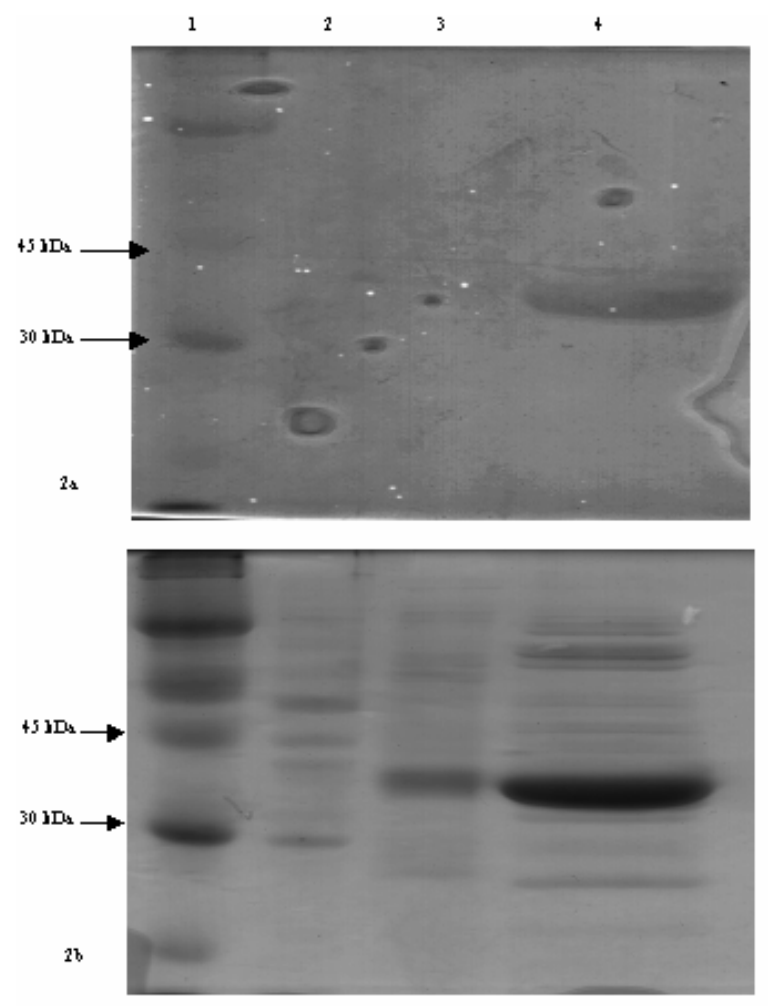

2 c

Fig. 2: (a) SDS-PAGE with HisProbe ${ }^{\mathrm{TM}}$ HRP; (b) Post staining of gel (a) with Coomassie blue; (c) Western Blot using HisProbe ${ }^{\mathrm{TM}}$ HRP. Lane 1: Rainbow Molecular Weight Marker, Lane 2: Promega Broad Range SDS-PAGE Standards, Lane 3: $15 \mu \mathrm{L}$ of E. coli lysate, Lane 4: $18 \mu \mathrm{g}$ of wild-type copro'gen oxidase

\section{REFERENCES}

1. Burnette, N.W., 1981. Western blotting: Electrophoretic transfer of proteins from sodium dodecyl sulfate-polyacrylamide gels to unmodified nitrocellulose and radiographic detection with antibody and radioiodinated protein A. J. Anal. Chem., 112: 195-203.

2. Medlock, A.E. and H.A. Dailey, 1996. Human coproporphyrinogen oxidase is not a metalloprotein. J. Biol. Chem., 271: 32507-32510.

3. Laemmli, U.K., 1970. Cleavage of structural proteins during assembly of the head of bacteriophage T4. Nature, 227: 680-685. 